\title{
A high plasma D-dimer level predicts poor prognosis in gynecological tumors in East Asia area: a systematic review and meta-analysis
}

\author{
Lei $\mathrm{Xu}^{1,2, *}$, Fan $\mathrm{He}^{3, *}$, Hongcai Wang${ }^{2}$, Bei Gao ${ }^{4}$, Huini $\mathrm{Wu}^{5}$ and Shuping Zhao ${ }^{1}$ \\ ${ }^{1}$ Gynecology Department, Qingdao University Affiliated Qingdao Women's and Children's Hospital, Qingdao, China \\ ${ }^{2}$ Gynecology Department, Maternal and Child Health Hospital of Zibo City, Shandong, China \\ ${ }^{3}$ Department of Molecular Oncology, H. Lee Moffitt Cancer Center \& Research Institute, Tampa, FL, USA \\ ${ }^{4}$ State Key Laboratory of Ophthalmology, Zhongshan Ophthalmic Center, Sun Yat-sen University, Guangzhou, China \\ ${ }^{5}$ Department of Biological Sciences, University of Illinois at Chicago, Chicago, IL, USA \\ "These authors have contributed equally to this work \\ Correspondence to: Shuping Zhao, email: zhaosp66@126.com
}

Keywords: D-dimer, gynecological tumors, meta-analysis, prognosis

Received: September 14, $2016 \quad$ Accepted: February 15, 2017

Published: May 16, 2017

Copyright: Xu et al. This is an open-access article distributed under the terms of the Creative Commons Attribution License 3.0 (CC BY 3.0), which permits unrestricted use, distribution, and reproduction in any medium, provided the original author and source are credited.

\section{ABSTRACT}

High pre-treatment plasma D-dimer levels have been reported as a factor associated with a poor prognosis in different types of malignancies, including pancreatic, gastric, colorectal, lung, and nasopharyngeal carcinoma. Here, we performed a meta-analysis to determine the association of plasma D-dimer levels and long term survival in gynecological cancers, including ovarian, cervical and endometrial carcinoma. We searched all eligible publications in PubMed and Web of Science Databases up to August 2016. Primary outcomes, including overall survival (OS), disease-free survival and hazard ratios (HR) of were extracted and analyzed. Heterogeneity and publication bias were also assessed. A total of 7 eligible studies with 1112 cases were included in this study and all included studies are conducted in East Asia area. We found that gynecological cancer patients with high D-dimer demonstrates a much lower 5-year survival rate than those with low D-dimer levels (OR 4.12, 95\% CI 3.04-5.58, $\mathrm{P}<0.00001)$. No significant heterogeneity is found $\left(\mathrm{I}^{2}=\right.$ $10 \%$; $P=0.35$ ). Importantly, pooled analysis showed that high plasma D-dimer levels are predictive of a shorter OS in gynecological cancers (HR 2.09, 95\% CI 1.59-2.74). No heterogeneity is observed $\left(I^{2}=5 \%, P=0.39\right)$. Additionally, a subgroup analysis of ovarian cancer is conducted. In conclusion, this meta-analysis showed that a high plasma D-dimer level predicts poor prognosis in gynecological tumors.

\section{INTRODUCTION}

Gynecological cancers, including ovarian, endometrial and cervical cancers, are major types of malignancies of genital system for women worldwide. In the United States, 95000 women are estimated to be diagnosed with gynecological cancers and 28800 will die of it in 2016 [1]. According to the GLOBOCAN database, the incidence and mortality of gynecological cancers are lower in East Asia than North America area.
However, because of the huge population in this area, it accounts for more than $20 \%$ of the incidence worldwide. There are about 750,000 new gynecological carcinoma cases worldwide and about 150,000 of them are from East Asia in 2012(20\%). Targeted therapeutics, such as EGFR inhibitors and other Serine/Threonine kinase inhibitors have shown limited efficacy in gynecological cancers and current therapies remain to be radical surgical tumor debulking plus platinum-based chemotherapy [2]. Thus, effective prognostic biomarkers are in great need 
to distinguish gynecological cancer patients who require more aggressive treatments.

Activation of coagulation and fibrinolysis have been frequently observed in many tumors [3]. Coagulation refers to the process of changing blood from liquid to gel, forming clots. Fibrinolysis is the degradation of fibrin to prevent blood clots from forming. D-dimer is two cross-linked D fragments of fibrin protein, the product of fibrin degradation [4]. In gynecological cancers, both coagulation and fibrinolysis systems are hyperactivated. Thus, the level of plasm D-dimer is also found elevated and associated with the formation of venous thromboembolism (VTE) [5]. Recently, elevated levels of D-dimer have shown predictive of survival in many malignancies, including lung, pancreatic, colorectal and breast cancer [6-9]. The relationship between high plasma D-dimer level and poor prognosis is also reported in gynecological cancers, including ovarian, cervical and endometrial cancers [10-16]. However, a systematic study is needed to address and to confirm the significance. The aim of this meta-analysis is to evaluate the prognostic significance of D-dimer across gynecological cancers, thereby providing references for clinical decisions.

\section{RESULTS}

\section{Literature search}

A total of 56 publications were identified in the initial literature search. Based on screening of titles or abstracts, 46 records were excluded. Full text articles were retrieved only for 10 publications and assessed for eligibility. 3 publications were excluded for insufficient data. Finally, 7 studies were included in this meta-analysis [10-16]. The process of identifying studies is shown as a flow chart in Figure 1. A total of 1112 patients were included and the characteristics of seven eligible studies are summarized in Tables 1 .

\section{Meta-analysis results}

Based on the Kaplan-Meier survival curves of these seven studies, we extracted and calculated 1-year, 3-year, and 5-year OS data. The estimated proportion of heterogeneity $\left(\mathrm{I}^{2}\right)$ between these seven studies was $10 \%$ $(\mathrm{P}=0.35)$ for 5-year OS rates. Therefore, no significant heterogeneity exists and a fixed-effect model was applied. However, heterogeneity exists in 1-year and 3-year OS rates and a random-effect model was applied. As Figure 2 demonstrates, patients with a high plasma D-dimer level had lower 1-year, 3-year, and 5-year OS rates than patients with a low plasma D-dimer level (80.13\% [371/463] vs $94.30 \%$ [612/649]; 56.30\% [261/463] vs 84.28\% [547/649]; 50.3\% [233/463] vs $81.2 \%$ [527/649]). OR and 95\% CI were all larger than 1, which supports that high plasma D-dimer level is a risk factor for patients. To further evaluate the prognostic effect of plasma D-dimer level in gynecological cancers, we used the multivariate HRs and their 95\% CI in these studies to calculate a combined HR, demonstrating that patients with a high plasma D-dimer level had a worse prognosis than patients with a low plasma D-dimer level (HR 2.09, 95\% CI 1.59-2.74, Figure 3). No heterogeneity was observed $\left(\mathrm{I}^{2}=5 \%, \mathrm{P}=0.39\right)$. Begg's funnel test was performed to estimate the publication bias of the literatures in this study. As shown in Figure 4, the shape of the Funnel plots showed no obvious evidence of asymmetry.

In addition, we understand that tumor development, treatment and prognosis among ovarian, cervical and endometrial carcinoma are quite different. A subgroup analysis of ovarian cancer for combined 1-year, 3-year, and 5-year OS rates revealed that high plasma D-dimer level remains to be a risk factor for ovarian cancer patient $(\mathrm{OR}=4.03,2.34,3.77$, respectively, Figure 5). We did not conduct subgroup analysis in cervical and endometrial cancers since the included studies are too few.

\section{DISCUSSION}

Plasma D-dimer, which is produced by the degradation of fibrin, has been widely recognized as a biomarker of hemostasis and fibrinolysis activation. It is playing an indispensable role in the determination of initial anticoagulant therapy in clinical practice. Recently, the elevation of plasma D-dimer level is observed in numeral clinical settings, including pregnancy, cardiovascular disease, cancer, post-surgery or trauma [17]. High D-dimer (DD) levels are report as a poor prognostic factor in patients with lung, prostate, cervical, and colorectal cancer. Recently, a large prospective study from the Vienna Cancer and Thrombosis study with 1178 cancer patients indicates that D-dimer associated with poor survival in solid cancer patients [18]. All the results demonstrated that D-dimer may play an important role in the tumorigenesis and prognosis.

In this study, we, for the first time, evaluated the prognostic significance of plasma D-dimer level in gynecological cancers. Patients with a high plasma D-dimer level have a worse 5-year prognosis than those with a low plasma D-dimer level (OR 4.12, 95\% CI 3.04-5.58, $\mathrm{P}<0.00001)$. We also demonstrated that high plasma D-dimer level is a risk factor of prognosis in gynecological cancers by calculating the pooled HR of high plasma D-dimer $(\mathrm{HR}=2.0995 \%$ CI 1.59-2.74, $\mathrm{P}<0.00001)$. We believe that this meta-analysis help definite the prognostic significance of plasma D-dimer level in gynecological tumors.

In terms of the mechanism by which D-dimer affect prognosis, classically, people believe that it is through the formation of VTE. H high D-dimer levels is found predictive of occurrence of VTE in patients with cancer [19]. VTE is a frequent complication of cancer, especially ovarian cancer $(1.2 \%)$, brain tumors $(1.2 \%)$, and pancreatic cancer $(1.1 \%)$, and ranks major causes of death in cancer patients [20]. However, recently large scaled prospective studies found that the association of 
high D-dimer levels with poor prognosis was independent of VTE in hematologic malignancies and solid tumors [18]. It raised the question that D-dimer might be able to affect prognosis through a VTE independent pathway. It has been suggested that the application of anticoagulation medicine, such as the low molecular weight heparins, unfractionated heparin and vitamin $\mathrm{K}$ antagonists, exerts an encouraging effect on survival of cancer patients [21]. In this respect, it is of great interest that to investigate the use of anticoagulation reagents in cancer patients with elevated D-dimer levels. However, further studies are needed to figure out the particular biological mechanism of D-dimer in cancer patients.
There are some limitations concerning this study. First, the number of included studies is not limited and all came from Asia, indicating the existence of ethnicity bias. In addition, the cutoff value to define high and low D-dimer levels in seven included studies vary, which increased the difficulty of doing a pooled study. We believe that a standard and well-defined testing system of plasma D-dimer is necessary in clinical practice. Last but not least, although no obvious publication bias was revealed in this study, potential bias might still existed since positive results were more likely to be published.

In a nutshell, an elevated plasma D-dimer level is significantly associated with poor response in

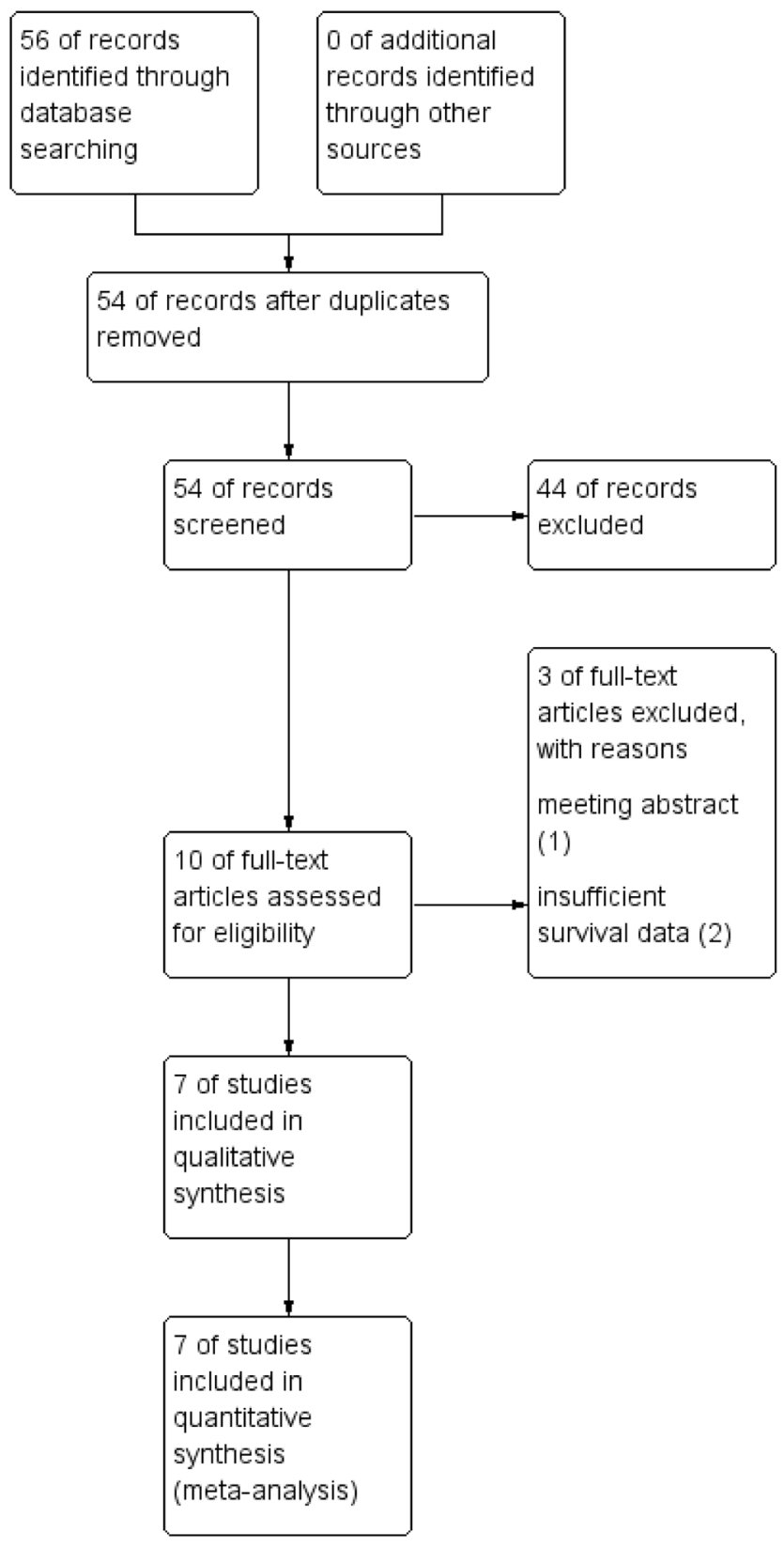

Figure 1: Flow diagram of search strategy in the meta-analysis. 
Table 1: Main characteristics of seven included studies in this meta-analysis

\begin{tabular}{lcccccccc}
\hline Study & Year & Tumor type & $\begin{array}{c}\text { Patient } \\
\text { source }\end{array}$ & $\begin{array}{c}\text { patient } \\
\text { number }\end{array}$ & $\begin{array}{c}\text { Multivariate/ } \\
\text { univariate }\end{array}$ & HR(95\%) & $\begin{array}{c}\text { P } \\
\text { value }\end{array}$ & $\begin{array}{c}\mathbf{6 0} \text { months } \\
\text { OS rates }\end{array}$ \\
\hline Sakurai & 2015 & Ovarian & Japan & 134 & Multivariate & $2.11(1.02-4.78)$ & 0.041 & $61.60 \%$ \\
Man & 2015 & Ovarian & China & 190 & Multivariate & $1.643(1.025-2.634)$ & 0.039 & $53.68 \%$ \\
Liu & 2015 & Ovarian & China & 125 & Multivariate & $1.901(1.021-3.540)$ & 0.043 & $53.60 \%$ \\
Nakamura & 2016 & Cervical & Japan & 129 & Multivariate & $2.33(1.121-5.504)$ & 0.043 & $73.64 \%$ \\
Luo & 2015 & Cervical & China & 296 & Multivariate & $2.148(1.035-4.457)$ & 0.036 & $67.9 \%$ \\
Nakamura & 2016 & Endometrial & Japan & 110 & Multivariate & $19.646(1.874-206.011)$ & 0.013 & $83.33 \%$ \\
Li & 2015 & Endometrial & China & 282 & Multivariate & $3.968(1.495-10.528)$ & $<0.05$ & $86.50 \%$ \\
\hline
\end{tabular}

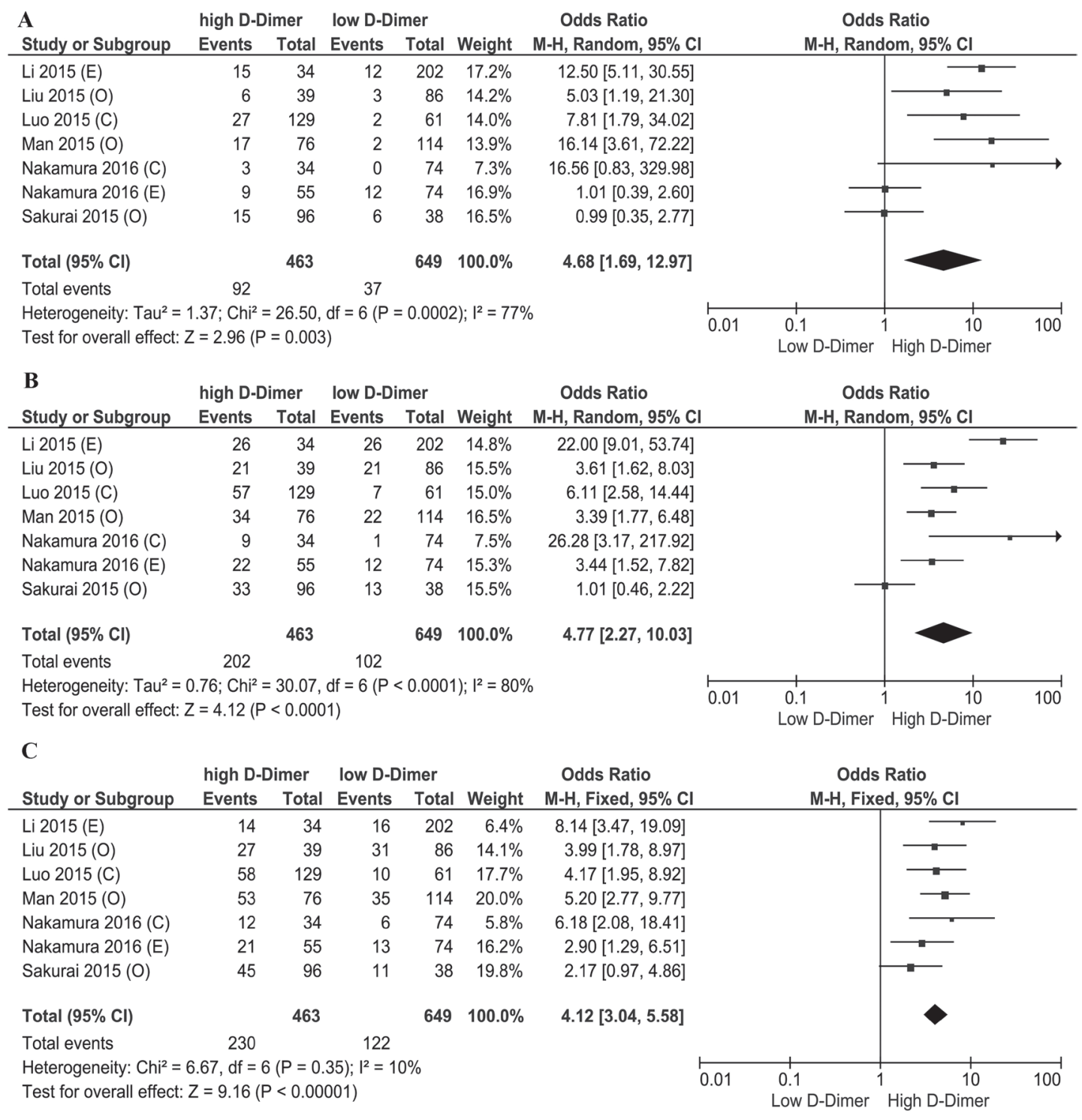

Figure 2: Forest plot of overall survival (OS) rates of high and low plasma D-dimer. (A) Forest plot of 1-year OS rates of high and low plasma D-dimer. (B) Forest plot of 3-year OS rates of high and low plasma D-dimer. (C) Forest plot of 5-year OS rates of high and low plasma D-dimer. C: cervical cancer, O: ovarian cancer, E: endometrial cancer. 


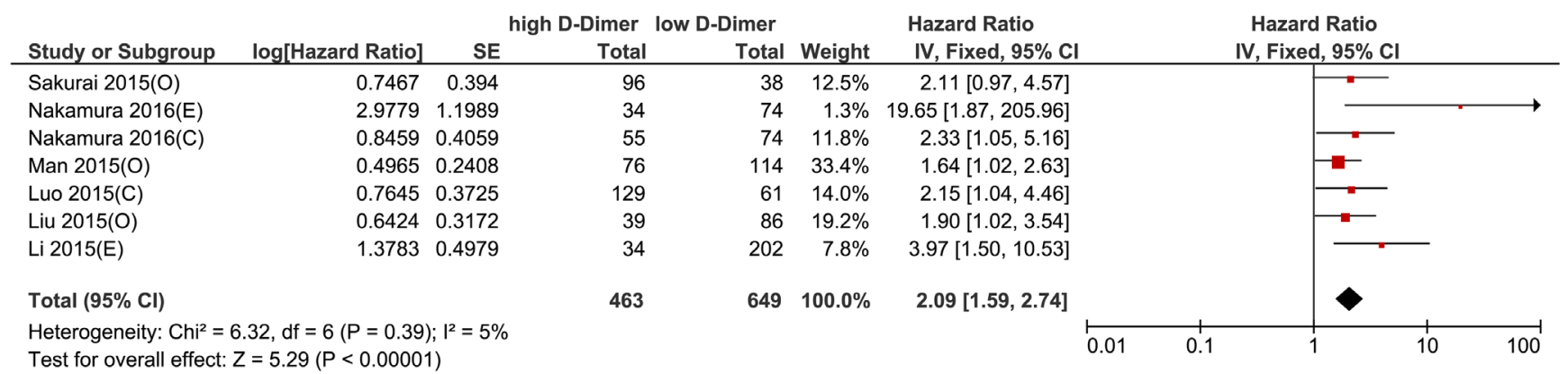

Figure 3: Forest plot of hazard ratio for the association of high plasma D-dimer level and OS; C: cervical cancer, O: ovarian cancer, E: endometrial cancer.
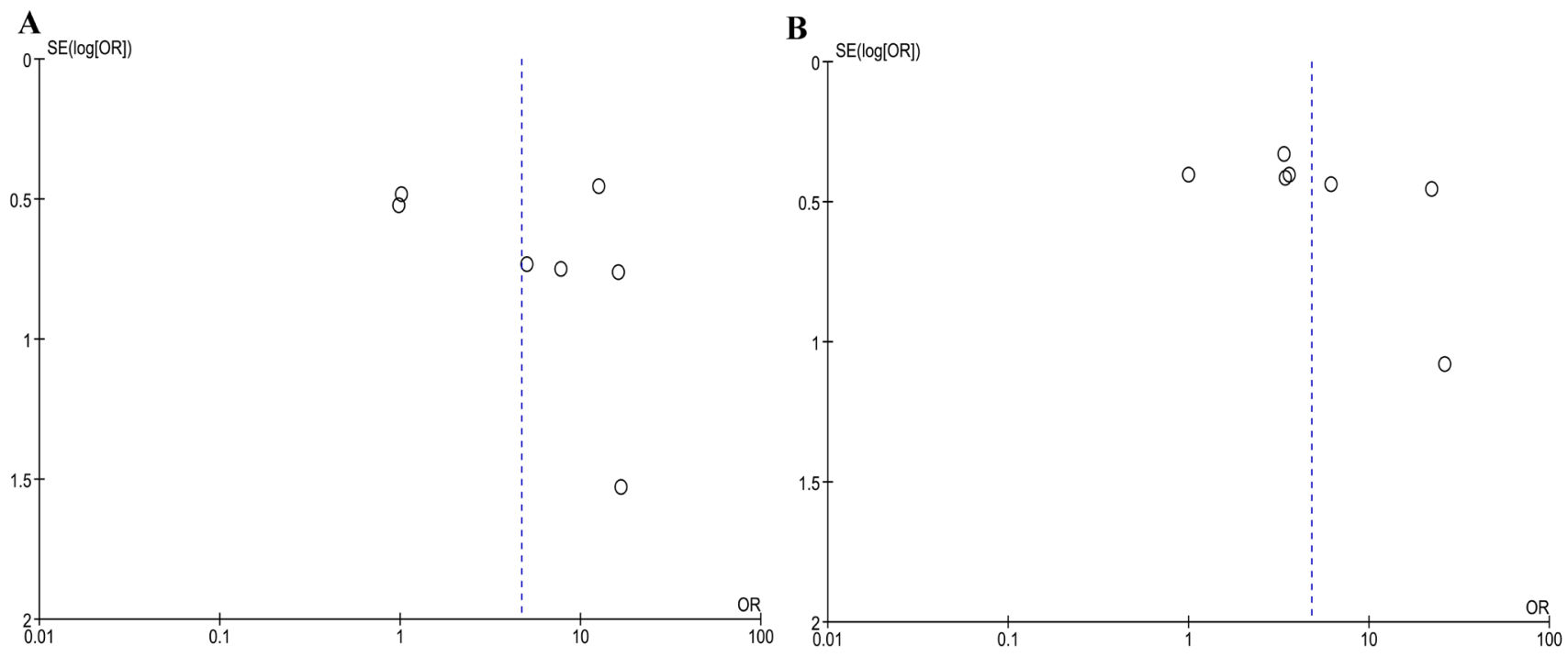

\section{C}

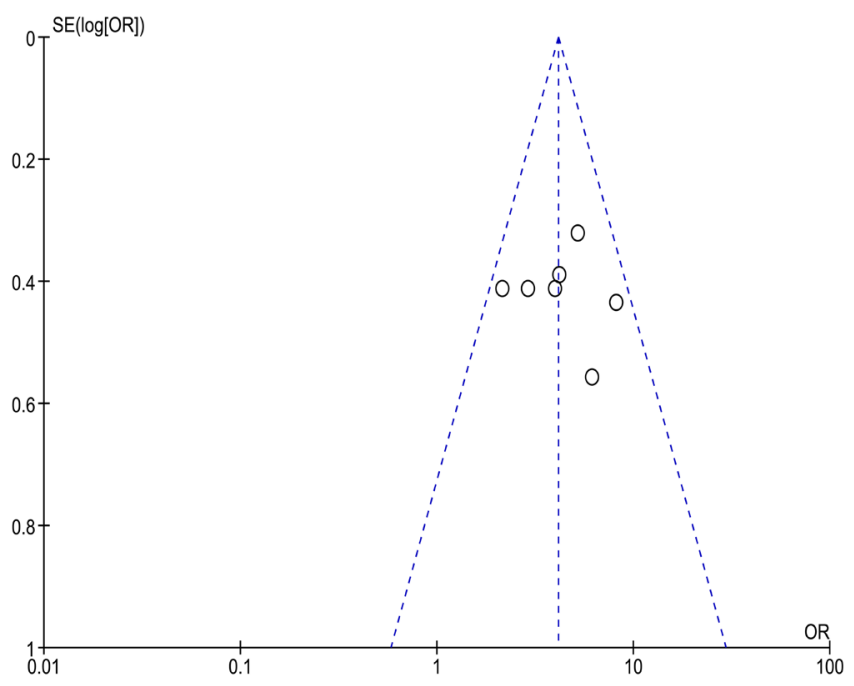

D

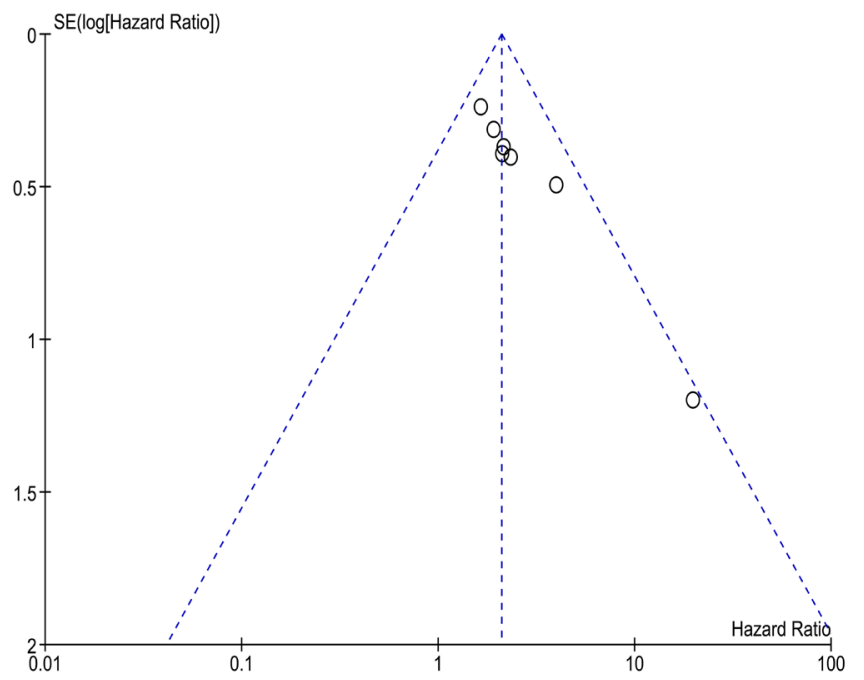

Figure 4: Begg's funnel plots of publication bias. (A) Meta-analysis of 1 year overall survival rates of high and low plasma D-dimer. (B) Meta-analysis of 3 year overall survival rates of high and low plasma D-dimer. (C) Meta-analysis of 5 year overall survival rates of high and low plasma D-dimer. (D) Meta-analysis of hazard ratio for the association of high plasma D-dimer level and OS. 


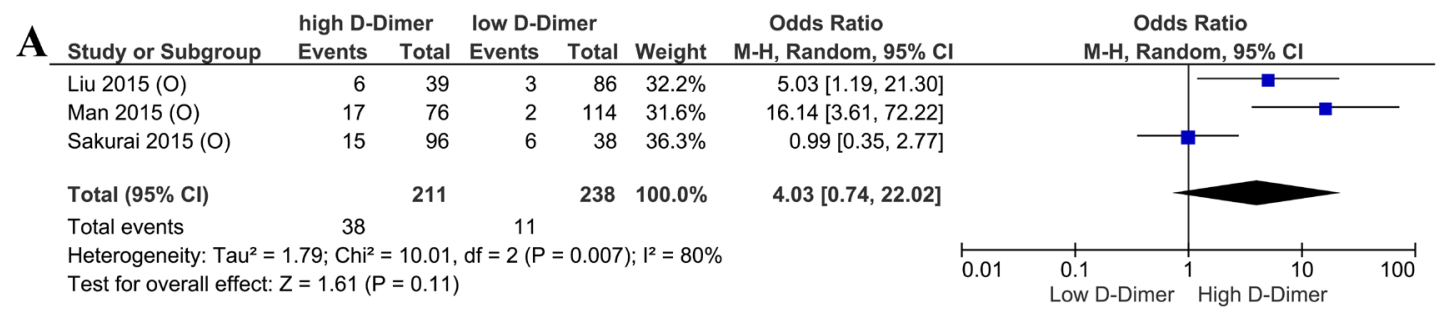

B

\begin{tabular}{|c|c|c|c|c|c|c|c|c|c|c|}
\hline \multirow[b]{2}{*}{ Study or Subgroup } & \multicolumn{2}{|c|}{ high D-Dimer } & \multicolumn{2}{|c|}{ low D-Dimer } & \multirow[b]{2}{*}{ Weight } & \multirow{2}{*}{$\begin{array}{l}\text { Odds Ratio } \\
\text { M-H, Random, } 95 \% \mathrm{CI}\end{array}$} & & \multirow{2}{*}{\multicolumn{2}{|c|}{$\begin{array}{c}\text { Odds Ratio } \\
\text { M-H, Random, } 95 \% \mathrm{Cl}\end{array}$}} & \\
\hline & Events & Total & Events & Total & & & & & & \\
\hline Liu 2015 (O) & 21 & 39 & 21 & 86 & $31.9 \%$ & $3.61[1.62,8.03]$ & & & & \\
\hline Man $2015(0)$ & 34 & 76 & 22 & 114 & $36.0 \%$ & $3.39[1.77,6.48]$ & & & & \\
\hline Sakurai $2015(0)$ & 33 & 96 & 13 & 38 & $32.1 \%$ & $1.01[0.46,2.22]$ & & & & \\
\hline Total $(95 \% \mathrm{Cl})$ & & 211 & & 238 & $100.0 \%$ & $2.34[1.07,5.14]$ & & & & \\
\hline Total events & 88 & & 56 & & & & & & & \\
\hline $\begin{array}{l}\text { Heterogeneity: Tau } \\
\text { Test for overall effec }\end{array}$ & $\begin{array}{l}34 ; \mathrm{Chi}^{2} \\
=2.12(\end{array}$ & $\begin{array}{l}=6.71, \mathrm{c} \\
=0.03)\end{array}$ & $\mathrm{df}=2(P=$ & $0.03) ;$ & $2=70 \%$ & & 0.01 & $\begin{array}{l}0.1 \\
\text { Low D-Dimer }\end{array}$ & $\begin{array}{c}10 \\
\text { High D-Dimer }\end{array}$ & 100 \\
\hline
\end{tabular}

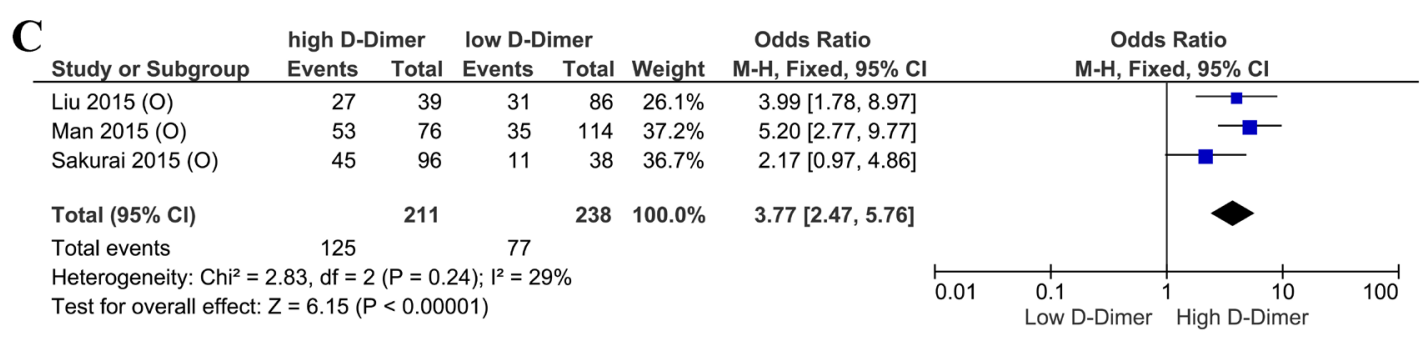

D

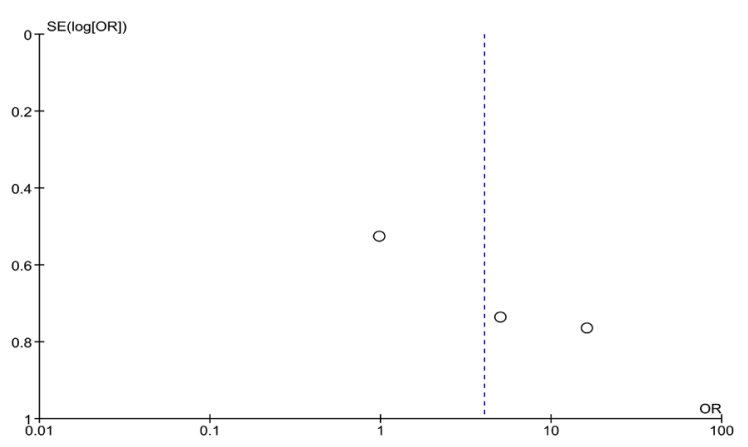

E

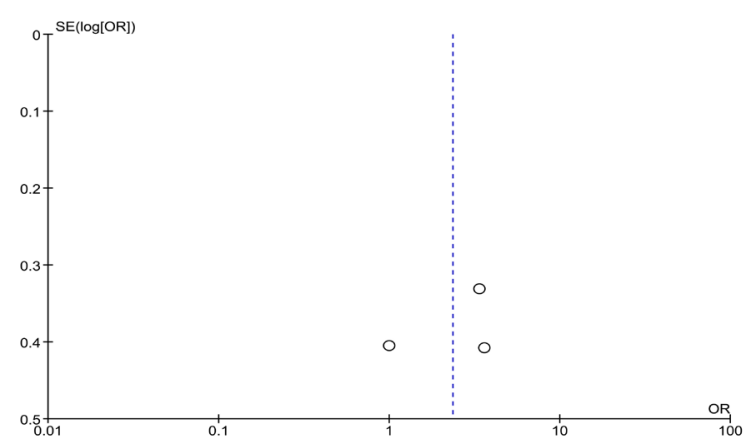

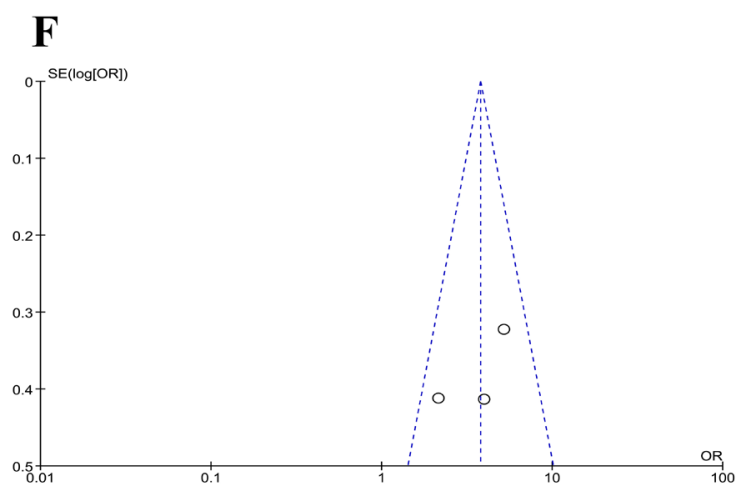

Figure 5: Subgroup analysis of ovarian cancer. (A) Forest plot of 1 year OS rates of high and low plasma D-dimer. (B) Forest plot of 3-year OS rates of high and low plasma D-dimer. (C) Forest plot of 5-year OS rates of high and low plasma D-dimer. (D) Begg's funnel plots of publication bias of 1-year OS rates of high and low plasma D-dimer. (E) Begg's funnel plots of publication bias of 3-year OS rates of high and low plasma D-dimer. (F) Begg's funnel plots of publication bias of 5-year OS rates of high and low plasma D-dimer. 
gynecological cancer patients. The plasma D-dimer level could be a prognostic biomarker of gynecological cancer patients.

\section{MATERIALS AND METHODS}

\section{Literature search}

Two investigators (Lei $\mathrm{Xu}$ and Bei Gao) independently searched eligible manuscripts in PubMed, Web of Science and China National Knowledge Infrastructure dating up to August, 2016. The following keywords and their combinations are applied in searching: "D dimer" or "D-dimer," and "ovarian cancer" or "cervical cancer" or "endometrial cancer" or "Endometrial carcinomas," and "prognosis" or "survival" from 20072016. The last search was performed on Sep 6, 2016. All of the eligible manuscripts and their references are retrieved for data extraction and analysis.

\section{Selection criteria}

Studies met the following criteria were collected in this meta-analysis: (1) all gynecological cancers, including ovarian cancer, cervical cancer and endometrial cancer were all histopathological diagnosed; (2) the level of D-dimer were detected prior to surgery; (3) OS, DFS and hazard ratios (HRs) with their $95 \%$ confidence interval (95\% CI) of OS/DFS of patients were evaluated in all studies; (4) if overlapping data were published in different reports by the same investigator, only the most complete one was included.

\section{Data extraction and quality assessment}

Two independent investigators (Fan $\mathrm{He}$ and Bei Gao) assess the quality of these publications using tools in Revman5.3 recommended by Cochrane Collaboration. It features five different bias, including selection, performance, detection, attrition, reporting and other potential sources of bias. Each category is ranked low risk, high risk or unclear For each study, following information were extracted: author, year of publication, country, number of patients, plasma D-dimer detection method, cutting off value of high D-dimer level, OS, DFS, 60 months survival rates and HR with its $95 \%$ CI. If the data couldn't be obtained directly, we calculated from KaplanMeier survival curve. Kaplan-Meier survival curves were imported into digitizing software (Engauge Digitizer 4.1, Free Software Foundation) to extract for 1-year and 3 -year survival rates. Two group members (Lei Xu and Huini $\mathrm{Wu}$ ) extract data independently to prevent typos and mistakes and disagreements are resolved by discussion to reach consensus.

\section{Statistical analysis}

All analysis were performed with Revman5.3 (The Nordic Cochrane Centre, the Cochrane Collaboration, Copenhagen, Denmark). Statistical heterogeneity between studies was determined by Cochran's Q test and Higgins I square. Heterogeneity was defined as $\mathrm{P}<0.1$ or $\mathrm{I}^{2}>50 \%$. In the absence of statistically significant heterogeneity, a fixed effects model is used to combine the data. Otherwise, a random effects model is applied. Publication bias is assessed by funnel plot with Revman5.3. Subgroup analysis is conducted by stratifying on tumor type. For all analyses, a two-sided $\mathrm{P}$ value less than 0.05 was considered statistically significant.

\section{CONFLICTS OF INTEREST}

The authors have no conflicts of interest to report.

\section{GRANT SUPPORT}

The authors have no grant to report.

\section{REFERENCES}

1. Siegel RL, Miller KD, Jemal A. Cancer statistics, 2016. CA Cancer J Clin. 2016; 66:7-30.

2. Vergote IB, Jimeno A, Joly F, Katsaros D, Coens C, Despierre E, Marth C, Hall M, Steer CB, Colombo N, Lesoin A, Casado A, Reinthaller A, et al. Randomized phase III study of erlotinib versus observation in patients with no evidence of disease progression after first-line platinbased chemotherapy for ovarian carcinoma: a European Organisation for Research and Treatment of CancerGynaecological Cancer Group, and Gynecologic Cancer Intergroup study. J Clin Oncol. 2014; 32:320-326.

3. Lyman GH, Khorana AA. Cancer, clots and consensus: new understanding of an old problem. J Clin Oncol. 2009; 27:4821-4826.

4. Adam SS, Key NS, Greenberg CS. D-dimer antigen: current concepts and future prospects. Blood. 2009; 113:2878-2887.

5. Ye S, Zhang W, Yang J, Cao D, Huang H, Wu M, Lang J, Shen K. Pattern of Venous Thromboembolism Occurrence in Gynecologic Malignancy: Incidence, Timing, and Distribution a 10-Year Retrospective Single-institutional Study. Medicine (Baltimore). 2015; 94:e2316.

6. Chen Y, Yu H, Wu C, Li J, Jiao S, Hu Y, Tao H, Wu B, Li A. Prognostic value of plasma D-dimer levels in patients with small-cell lung cancer. Biomed Pharmacother. 2016; 81:210-217.

7. Stender MT, Larsen AC, Sall M, Thorlacius-Ussing O. D-Dimer predicts prognosis and non-resectability in patients with pancreatic cancer: a prospective cohort study. Blood Coagul Fibrinolysis. 2016; 27:597-601. 
8. Zhu L, Liu B, Zhao Y, Liu L, Yang C, Yang Y, Zhong H. High levels of D-dimer correlated with disease status and poor prognosis of inoperable metastatic colorectal cancer patients treated with bevacizumab. J Cancer Res Ther. 2014; 10:246-251.

9. Liu YL, Lu Q, Liang JW, Xia Y, Zhang W, Hu BQ, Shang FF, Ji YR, Wang J, Wang Q, Liang B. High plasma fibrinogen is correlated with poor response to trastuzumab treatment in HER2 positive breast cancer. Medicine (Baltimore). 2015; 94:e481.

10. Nakamura K, Nakayama K, Ishikawa M, Katagiri H, Minamoto T, Ishibashi T, Ishikawa N, Sato E, Sanuki K, Yamashita H, Komatsu-Fujii T, Kyo S. High Pretreatment Plasma D-Dimer Level as a Potential Prognostic Biomarker for Cervical Carcinoma. Anticancer Res. 2016; 36:2933-2938.

11. Luo YL, Chi PD, Zheng X, Zhang L, Wang XP, Chen H. Preoperative D-dimers as an independent prognostic marker in cervical carcinoma. Tumour Biol. 2015; 36:8903-8911.

12. Nakamura K, Nakayama K, Ishikawa M, Katagiri H, Minamoto T, Ishibashi T, Ishikawa N, Sato E, Sanuki K, Yamashita H, Komatsu-Fujii T, Kyo S. High pretreatment plasma D-dimer levels are related to shorter overall survival in endometrial carcinoma. Eur J Obstet Gynecol Reprod Biol. 2016; 201:89-93.

13. Li J, Lin J, Luo Y, Kuang M, Liu Y. Multivariate Analysis of Prognostic Biomarkers in Surgically Treated Endometrial Cancer. PLoS One. 2015; 10:e0130640.

14. Sakurai M, Satoh T, Matsumoto K, Michikami H, Nakamura Y, Nakao S, Ochi H, Onuki M, Minaguchi T, Yoshikawa H. High Pretreatment Plasma D-dimer Levels Are Associated With Poor Prognosis in Patients With Ovarian Cancer Independently of Venous Thromboembolism and Tumor Extension. Int J Gynecol Cancer. 2015; 25:593-598.
15. Liu P, Wang Y, Tong L, Xu Y, Zhang W, Guo Z, Ni H. Elevated preoperative plasma D-dimer level is a useful predictor of chemoresistance and poor disease outcome for serous ovarian cancer patients. Cancer Chemother Pharmacol. 2015; 76:1163-1171.

16. Man YN, Wang YN, Hao J, Liu X, Liu C, Zhu C, Wu XZ. Pretreatment plasma D-dimer, fibrinogen, and platelet levels significantly impact prognosis in patients with epithelial ovarian cancer independently of venous thromboembolism. Int J Gynecol Cancer. 2015; 25:24-32.

17. Lippi G, Franchini M, Targher G, Favaloro EJ. Help me, Doctor! My D-dimer is raised. Ann Med. 2008; 40:594-605.

18. Ay C, Dunkler D, Pirker R, Thaler J, Quehenberger P, Wagner O, Zielinski C, Pabinger I. High D-dimer levels are associated with poor prognosis in cancer patients. Haematologica. 2012; 97:1158-1164.

19. Ay C, Vormittag R, Dunkler D, Simanek R, Chiriac AL, Drach J, Quehenberger P, Wagner O, Zielinski C, Pabinger I. D-dimer and prothrombin fragment $1+2$ predict venous thromboembolism in patients with cancer: results from the Vienna Cancer and Thrombosis Study. J Clin Oncol. 2009; 27:4124-4129.

20. Levitan N, Dowlati A, Remick SC, Tahsildar HI, Sivinski LD, Beyth R, Rimm AA. Rates of initial and recurrent thromboembolic disease among patients with malignancy versus those without malignancy. Risk analysis using Medicare claims data. Medicine (Baltimore). 1999; 78:285-291.

21. Kuderer NM, Ortel TL, Francis CW. Impact of venous thromboembolism and anticoagulation on cancer and cancer survival. J Clin Oncol. 2009; 27:4902-4911. 